\title{
Articles You Might Have Missed
}

\section{Rachel S. Weiselberg}

Published online: 15 October 2011

(C) American College of Medical Toxicology 2011

Keywords Cocaine-induced chest pain - Acute lung injury . Contrast-induced nephropathy $\cdot$ High-dose insulin glucose therapy $\cdot$ Intravenous fat emulsion

Chang AM, Walsh KM, Shofer FS, et al. Relationship between cocaine use and coronary artery disease in patients with symptoms consistent with an acute coronary syndrome. Acad Emerg Med 2011; 18: 1-9

Background: Cocaine complications are a common cause of emergency department visits. Of cocaine-related visits, $40 \%$ are for chest pain. Cocaine has several physiological mechanisms that possibly accelerate the development of coronary artery disease (CAD) and previous clinical series suggest a higherthan-expected prevalence of CAD in younger individuals.

Research question: In chest pain patients with low- to intermediate-risk for an acute coronary syndrome, is there a significant relationship between cocaine and CAD?

Methods: This is a retrospective cross-sectional analysis of patients who received coronary computerized tomographic angiography (CTA) during an evaluation of chest pain and had apparent low to intermediate risk of CAD. The main outcome was the presence of CAD $(\leq 25 \%, 25-49 \%,>50 \%)$. Cocaine use was categorized as no use, single use, or repetitive use. If cocaine use was identified, it was also categorized as recent (within

This article review has not been presented previously.

R. S. Weiselberg $(\bowtie)$

Department of Emergency Medicine,

North Shore University Hospital,

Manhasset, NY 11030, USA

e-mail: rweiselberg@gmail.com
1 week of presentation or positive urine screen) versus not recent. Chi-square, Fisher's exact and Student's t tests were used with an adjusted relative risk to assess the relationship between $\mathrm{CAD}$ and cocaine use.

Results: Of the 912 patient included in this review, 155 were repetitive cocaine users, 74 of whom had recent use by history or urine screen. There was no significant difference in the finding of CAD between repetitive or recent cocaine users versus nonusers. This was irrespective of whether CAD was defined by the size of the coronary lesion or the calcium score. Cocaine users were twice as likely to use tobacco.

Conclusion: Cocaine use does not increase the likelihood for CAD in low- to intermediate-risk patients presenting to the emergency department with chest pain.

Critique: Positives to this study included a much larger population than previous work, significant effort at identifying cocaine use, and appropriate analysis. On the other hand, selection of patients for CTA may not have been uniform and therefore influenced results: The decision to have a patient undergo coronary CTA was made at the discretion of the treating physician. We do not know if there was bias for or against imaging patients when cocaine use was suspected. The authors relied on historical presence of CAD risk factors such as hypercholesterolism and tobacco use. Additionally, as noted in the limitation section, duration of cocaine use was not studied.

The study results generate some intriguing questions. The average age in the study is 46 years, but for patients with CAD $>50 \%$ is 53 years. It would be interesting to see the data on the average age of the cocaine user versus the nonuser with $\mathrm{CAD}>50 \%$. It is also interesting that, despite having twice as many smokers in the cocaine group, there 
was no increased prevalence of CAD compared to nonusers. This might be due to under-reported tobacco use in noncocaine users or lack of data concerning chronicity of cocaine use.

Implication for toxicologists: Although this study was well done, it is not robust enough to completely discount cocaine as a risk factor for CAD. Further studies are needed to understand the impact of acuity and chronicity of cocaine abuse as potential modifiers of the prevalence of CAD.

Harvey M, Cave G, Lahner D, et al. Insulin versus lipid emulsion in a rabbit model of severe propranolol toxicity: A pilot study. Crit Care Res Practice 2011; 2011; 1-7

Background: The management of beta-blocker toxicity is challenging. Both high-dose insulin-glucose (ING) therapy and intravenous lipid emulsion (ILE) were beneficial in multiple animal models and limited human case reports. These two antidotal therapies have not been tested head to head.

Research question: In propranolol toxicity, how does the hemodynamic effect of ING therapy compare to ILE?

Methods: Using a rabbit model, propranolol toxicity was achieved by instilling propranolol directly into the duodenum, followed by an intravenous bolus and continuous infusion with a goal mean arterial pressure (MAP) of 50\% of baseline. Ten animals were randomized to receive either $20 \%$ ILE at $10 \mathrm{~mL} / \mathrm{kg}$ or 3 units $/ \mathrm{kg}$ insulin with $0.5 \mathrm{~g} / \mathrm{kg}$ glucose. Heart rate (HR) and MAP were monitored for $1 \mathrm{~h}$ and rate pressure products $(\mathrm{RPP} ; \mathrm{RPP}=\mathrm{HR} \times \mathrm{MAP})$ were calculated. In the event of cardiac collapse, adrenaline boluses and chest compressions were begun, along with a second bolus of the corresponding study intervention. If, after $10 \mathrm{~min}$, they did not have a return of spontaneous circulation, they were crossed over to the other treatment arm. The main outcome was hemodynamic parameters compared with two-way repeated measures analysis of variance. Survival was a secondary outcome.

Results: There was no difference between the MAP and HR of the two groups. The RPP was significantly higher in the ING group at the end of the protocol. Cardiac collapse occurred in $1 / 5$ ING rabbits and $3 / 5$ in the ILE group and none of these animals survived despite additional antidote treatment.

Conclusion: In a rabbit model of propranolol toxicity, ING therapy produced a significant increase in the RPP over ILE.

Critique: This comparative study is an important step in elucidating the optimal antidote for propranolol toxicity. As this was a pilot study, the small sample size may have led to type II errors in the analysis, especially regarding survival effect. Additionally, very aggressive initial doses of ILE and insulin were used and it is unclear how this aspect of the study may impact translation to clinical care. The study may be improved by monitoring metabolic parameters such as glucose, lactate, and oxygen saturation that might yield data pertinent to effectiveness and safety of these antidotes. Implication for toxicologists: For beta-blocker toxicity, ING is already recognized as an important therapy by toxicologists. ILE therapy is an accepted antidote for local anesthetic toxicity and is gaining popularity for use in cardiac collapse due to non-anesthetic lipophilic xenobiotics. This pilot study highlights the need for further comparative studies between ILE and other more established antidotes.

Ueda H, Yamada T, Masuda M, et al. Prevention of contrast-induced nephropathy by bolus injection of sodium bicarbonate in patients with chronic kidney disease undergoing emergent coronary procedures. Am J Cardiol 2011; 107: 1163-7

Background: Sodium bicarbonate has been given prophylactically to high-risk patients for contrast-induced nephropathy (CIN). One hour of pretreatment is suggested, which is less than ideal in preparing for emergency cardiac catheterization procedures. There is limited data in the setting of chronic kidney disease (CKD).

Research question: Can bolus sodium bicarbonate administered less than $1 \mathrm{~h}$ prior to emergent coronary procedures prevent $\mathrm{CIN}$ in patients with CKD?

Methods: This was a prospective trial of adults with CKD (creatinine $>1.1 \mathrm{mg} / \mathrm{dL}$ or estimated GFR $<60 \mathrm{~mL} / \mathrm{min}$ ) undergoing emergency coronary procedures within $60 \mathrm{~min}$ of presentation. Patients were randomized to receive a bolus of sodium bicarbonate $(154 \mathrm{mEq})$ or sodium chloride $(0.5 \mathrm{ml} / \mathrm{kg})$ prior to contrast. Both groups received sodium bicarbonate infusion for $6 \mathrm{~h}$ after contrast infusion was complete. Serum creatinine and blood urea nitrogen levels were checked on arrival, post-procedure days 2 and 3, and on discharge. CIN was defined as an increase of creatinine of $0.5 \mathrm{mg} / \mathrm{dL}$ or $25 \%$ above baseline by day 2 postprocedure. Comparison of baseline and post-procedure variables was made with the $t$ test, chi-square test, or Fisher's exact test. Independent factors associated with CIN were determined by multivariate logistic regression.

Results: Fifty-nine patients were included out of a targeted 80. Early analysis revealed that sodium bicarbonate substantially decreased the incidence of CIN, thus, enrollment was halted early due to ethical concerns. Pretreatment was administered 2-21 min prior to contrast. The sodium chloride group had significantly higher creatinine levels on day 2 and at discharge over baseline and $28 \%$ developed CIN. There was no significant creatinine change overall for the sodium 
bicarbonate group and the incidence of CIN was $3 \%$. No patients developed renal failure requiring dialysis. Conclusion: A single sodium bicarbonate bolus given $<30$ min prior to emergent contrast administration followed by bicarbonate infusion can prevent CIN in patients with baseline CKD.

Critique: The current study is a modification of a previous trial (Masuda et al. Am J Cardiol 2007; 100:781-6) where patients received either bicarbonate or saline pre- and postemergent contrast administration. This study suggests that pre-contrast bicarbonate is important in preventing CIN. The safety profile appears acceptable without any patients developing heart failure during the treatment period. However, several patients in both groups developed congestive heart failure "sometime" after the protocol. The authors looked at a population with at least stage 3 $\mathrm{CKD}$, the target population of the other bicarbonate studies. It would be interesting to see if there was a difference between patients with stage 3 versus stage 4 CKD.

Implication for toxicologists: While the single bolus appears effective in this, and previous studies, to prevent $\mathrm{CIN}$, it would be interesting to see a study comparing the efficacy of bicarbonate bolus to 1-h pre-contrast drip. Additionally, there are many other urgent contrast procedures (i.e., emergency department CT scans), where bicarbonate bolus may be useful if the patient is high risk for CIN.

Lange M, Hamahata A, Traber DL, et al. Preclinical evaluation of epinephrine nebulization to reduce airway hyperemia and improve oxygenation after smoke inhalation injury. Crit Care Med 2011; 39: 718-24

Background: Smoke inhalation can lead to significant acute lung injury (ALI). ALI results from a combination of factors including bronchospasm, hyperemia, edema, and the inflammatory response. Epinephrine (EPI) may be a useful treatment due to its vasoconstrictor and a bronchodilator properties.

Research question: Can nebulized epinephrine attenuate acute lung injury due to smoke inhalation in sheep?

Methods: This was a prospective sheep study. A preliminary trial establish that $4 \mathrm{mg}$ EPI was the optimal dose for efficacy and safety. ALI was induced via a standard protocol of exposure to cotton smoke. Animals were randomized to either nebulized epinephrine $4 \mathrm{mg}$, nebulized saline, or non-injured, non-treated controls. Ventilators were set to a tidal volume of $12-15 \mathrm{~mL} / \mathrm{kg}$, positive end-expiratory pressure of $5 \mathrm{~cm} \mathrm{H}_{2} \mathrm{O}$, a $\mathrm{F}_{\mathrm{I}} \mathrm{O}_{2}$ of $100 \%$ and a rate set to maintain $\mathrm{PaCO}_{2}$ within $5 \mathrm{mmHg}$ of baseline. Authors monitored pulmonary hemodynamics, pulmonary functions, inflammatory mediators, catecholamine levels, and histological changes.

Results: Smoke exposure increased microvascular blood flow, histological obstruction scores, weight/dry weight ratio, malondialdehyde levels and pulmonary shunt fraction, while it decreased the $\mathrm{PaO}_{2} / \mathrm{F}_{\mathrm{I}} \mathrm{O}_{2}$ ratio to less than $200 \mathrm{mmHg}$. Intermittent EPI attenuated all parameters of ALI compared to saline except histological obstruction score. This was accomplished without significant elevations of catecholamines or changes in liver and renal function. EPI transiently elevated cardiac index and serum lactate.

Conclusion: Nebulized epinephrine mitigates pulmonary injury secondary to smoke inhalation in a sheep model.

Critique: The prevention of ALI with epinephrine is nicely demonstrated by this well-done animal study. The tidal volumes used for the sheep, however, have been shown to induce volutrauma in sheep (Ballard-Croft et al. J Surg Res 2010; 164: e155-62), so it is unclear how much this variable contributed to the overall lung injury. While the study showed beneficial treatment effect with minimal adverse effect, the animals were otherwise healthy. The apparent safety may not be translated to animals or humans with significant preexisting medical conditions.

Implication for toxicologists: The study result demonstrates that a familiar, inexpensive, and readily available drug is very promising as a treatment for ALI due to smoke inhalation. More data is needed prior to widely instituting this intervention.

Conflict of interest There are no sources of funding or conflicts of interest to disclose. 